\title{
DNA methylation is related to the occurrence of breast cancer and is not affected by culture conditions
}

\author{
SHIBAO WANG ${ }^{1}$, YINGHUI HUANG ${ }^{2}$, XUPENG MU $^{2}$, \\ TIANYANG $\mathrm{QI}^{2}$, SHA QIAO ${ }^{1}$, ZHENXIA LU ${ }^{1}$ and HONGJUN LI ${ }^{3}$ \\ ${ }^{1}$ Department of Oncology and Hematology; ${ }^{2}$ Science Research Center; ${ }^{3}$ Physical Examination Center, \\ China-Japan Union Hospital, Jilin University, Changchun, Jilin 130033, P.R. China
}

Received April 26, 2016; Accepted September 6, 2017

DOI: $10.3892 / \mathrm{mmr} .2018 .8735$

\begin{abstract}
The present study aimed to explore the relationship between DNA methylation and breast cancer under different cell culture conditions. MCF-7 breast cancer cells were cultured in two-dimensional (2D), three-dimensional (3D) and orthotopic transplantation (Ti) adhesion substrates. Principal component analysis (PCA) was used for global visualization of these three samples. The methylation status of $\mathrm{CpG}$ sites was examined by unsupervised clustering analysis. Scatter plots and histograms were constructed from the mean $\beta$-values from 3D vs. 2D, 3D vs. Ti and Ti vs. 2D analysis. In addition, analyses of Gene Ontology (GO) terms and Kyoto Encyclopedia of Genes and Genomes (KEGG) pathways were conducted to explore the putative biological functions in which mutL homolog $(M L H)$, phosphatase and tensin homolog $(P T E N)$, runt-related transcription factor $(R U N X)$, Ras association domain family (RASSF), cadherin 1 (CDH1), O-6-methylguanine-DNA methyltransferase (MGMT) and P16 may serve a role. Quantitative methylation-specific polymerase chain reaction (QMSP) was performed to determine the influence of culturing conditions on important gene expression. Results from PCA analysis indicated that the three samples were closely connected with each other. Venn diagrams revealed that certain differential methylation positions were common among the three sample groups, and $116 \mathrm{CpG}$ positions were identified that appeared to be hypermethylated. The methylation patterns were more similar between 3D vs. 2D cultures compared with those between $3 \mathrm{D}$ vs. Ti or between Ti vs. 2D. Results of GO term and KEGG pathway analyses indicated that genes were enriched in four pathways, including transporter activity and G-protein coupled receptor activity. In addition, QMSP analysis identified no
\end{abstract}

Correspondence to: Dr Hongjun Li, Physical Examination Center, China-Japan Union Hospital, Jilin University, 126 Xiantai Street, Changchun, Jilin 130033, P.R. China

E-mail: lihongjun1030@126.com

Key words: breast cancer, DNA methylation, $\mathrm{CpG}$, culture conditions notable differences in the methylation status of $M L H, P T E N$, RUNX, RASSF, CDH1, MGMT and P16 under 2D, 3D and Ti culture conditions. In conclusion, abnormal DNA methylation is related with breast cancer, and the methylation status did not change in breast cancer cells cultured in different conditions.

\section{Introduction}

Breast cancer is a heterogeneous disease and is the most frequently diagnosed type of cancer in women, with an estimated 1.38 million new cases per year worldwide (1). Breast cancer patients with the same stage of disease may have different treatment responses and overall outcome (2). In breast cancer, epigenetic modifications are often noticed, including aberrant DNA hypermethylation (3). As previously reported, DNA methylation often occurs at carbon-5 of cytosine residues in $\mathrm{CpG}$ dinucleotides (4). Methylation changes in $\mathrm{CpG}$ islands (CGI) and $\mathrm{CpG}$ shores (low CpG density areas $\sim 2 \mathrm{~kb}$ close to CGI) affect gene expression and reprogramming $(5,6)$. In general, hypermethylation of $\mathrm{CpG}$ sites at tumor suppressor gene promoters and hypomethylation at oncogene promoters is thought to be involved in cancer (7). CpG islands are often observed in the promoter region and serve a crucial role in regulating key cellular functions (8). Hypermethylation in gene promoters seems to be an early event in carcinogenesis and the number of genes affected increases with breast cancer progression (9). DNA methylation has long been considered a key regulator of gene expression. The genetic basis of gene expression has been investigated across tissues and populations (10). It plays an important regulatory role in eukaryotic genomes. Alterations in methylation can affect transcription and phenotypic variation (11). A previous study indicated that genetic variation may have a substantial impact on local methylation patterns (12).

The culture of mammalian cells in vitro provides a defined platform for investigating cell and tissue physiology and pathophysiology outside of the organism (13). Traditionally, this has been done by culturing single cell populations on two-dimensional (2D) substrates such as tissue culture polystyrene (TCPS) or the surface of tissue analogs (14). Experiments with these 2D cell constructs have provided the base for preliminary interpretation of complex biological phenomena, including molecular biology, stem 
cell differentiation, and tissue morphogenesis. Furthermore, $2 \mathrm{D}$ experiments have given rise to seminal findings in the dynamic association between cell function and interactions with the cellular microenvironment (15). However, previous work demonstrated that cells often exhibit unnatural behavior when excised from native three-dimensional (3D) tissues and confined as a monolayer (16). 2D culture is technically easier and simple, but there is a lack of a natural microenvironment. Tumor cell growth is easily affected by internal and external environments and the cost of 2D culture is high. 3D culture can simulate the complex growth environment in the body, tumor tissue complex signal transduction pathways and the formation of new blood vessels (17). It is similar to Ti culture, it can simulate cell complex growth environment and is easy to establish, but it is still different from the internal growth environment for tumor cells. However, whether changes in DNA methylation state are influenced by the cell culture method in breast cancer is still unknown.

In the present study, it was aimed to investigate the influence of breast cancer cell culture method on DNA methylation state. Results indicated that methylation status did not change in breast cancer cells cultured under either $2 \mathrm{D}, 3 \mathrm{D}$ or $\mathrm{Ti}$ conditions.

\section{Materials and methods}

Cell culture. The breast cancer cell line MCF-7 was obtained from Ruijin Hospital, Affiliated to Shanghai Jiaotong University (Shanghai, China). Cells were cultured in Dulbecco's modified Eagle's medium (Invitrogen; Thermo Fisher Scientific, Inc. Waltham, MA, USA) supplemented with $10 \%$ fetal bovine serum (Sigma-Aldrich; Merck KGaA, Darmstadt, Germany). Subsequently, cells were cultured in 2D (density, $60,000 / \mathrm{cm}^{2}$ ), 3D (density, $70,000 / \mathrm{cm}^{2}$ ) and $\mathrm{Ti}$ adhesion substrates (density, $60,000 / \mathrm{cm}^{2}$ ) in $5 \% \mathrm{CO}_{2}$ at $37^{\circ} \mathrm{C}$. For $2 \mathrm{D}$ substrates, $2 \%$ alginate solution was mixed with calcium sulfate (Sigma-Aldrich; Merck $\mathrm{KGaA}$ ) and cast between glass plates, from which topographically flat hydrogel disks were punched out. Excess calcium was leached out by changing the medium every day for 4 days prior to seeding. The 3D adhesion substrate was prepared by suspending cells in $2 \%$ alginate solution. A custom-designed encapsulation unit was used, and the alginate/cell suspension was extruded into an isotonic $5.0 \%$ (w/v) $\mathrm{CaCl}_{2}$ cross-linking solution (Sigma-Aldrich; Merck KGaA); the generated beads were washed with PBS to remove excess $\mathrm{Ca}^{2+}$ and were dynamically cultured in spinner flasks (Bellco Glass, Inc., Vineland, NJ, USA). For Ti culture, cells were added to in situ culture flasks with $3 \mathrm{ml}$ Active Messages 2.0 (AM-II; Gibco; Thermo Fisher Scientific, Inc.). Finally, flasks were placed into a humidified cell incubator containing $5 \% \mathrm{CO}_{2}$ at $37^{\circ} \mathrm{C}$ for 5-7 days.

Total RNA isolation and chip genome-wide methylation detection. The RNeasy mini kit (Qiagen, Inc., Valencia, CA, USA) was used to isolate total RNA from $6 \times 10^{3} / \mathrm{ml}$ cells according to the manufacturer's protocol. Subsequently, the DNA was performed whole-genome detection by DNA methylation 450k BeadChips (Illumina Inc., San Diego, CA, USA). The extracted genomic DNA was processed by hydrosulfite transformation using EZ DNA Methylation kit (Zymo Research Corp., Irvine, CA, USA). Bisulfite-converted genomic DNA is amplified using locus-specific PCR primers flanking an oligonucleotide probe with a 5 ' fluorescent reporter dye (6FAM) and a 3 ' quencher dye (TAMRA) (18). Amplified DNA was cut into segments by DNA restriction endonucleases at $37^{\circ} \mathrm{C}$, and the DNA fragments were precipitated by isopropanol. DNA pellet after centrifugation at $12,000 \mathrm{~g}$, at room temperature for $5 \mathrm{~min}$ was resuspended in buffer RA1, and the resuspended DNA samples with concentration of $2 \times 10^{3} \mu \mathrm{g} / \mu 1$ were dispersed on BeadChip chips and Illumina Human HT-12 V4.0 expression BeadChip (Illumina, Inc.) was used for hybridization at $37^{\circ} \mathrm{C}$ (19). Arrays were scanned on the Illumina iScan system, and raw data was imported and analyzed with the BeadStudio software (version 3.1.3.0 Illumina, Inc). Prior to use, Illumina data we reserved on the basis of the MIAME guidelines in Gene Expression Omnibus database (www.ncbi.nlm.nih.gov/geo/).

DNA bisulfite modification. A total of $1.5 \mu \mathrm{g}$ DNA was denatured in $50 \mu \mathrm{l}$ of $0.2 \mathrm{M} \mathrm{NaOH}$ at $37^{\circ} \mathrm{C}$ for $10 \mathrm{~min}$. Then, $30 \mu \mathrm{l}$ of freshly prepared $10 \mathrm{mM}$ hydroquinone (Sigma-Aldrich; Merck $\mathrm{KGaA}$ ) and $520 \mu \mathrm{l}$ of $3 \mathrm{M}$ sodium bisulfite (Sigma; Merck $\mathrm{KGaA}$ ) at pH 5.0 were added and mixed. The samples were overlaid with mineral oil to prevent evaporation and incubated at $50^{\circ} \mathrm{C}$ for $16 \mathrm{~h}$. The bisulfite-treated DNA was isolated using Wizard DNA Clean-Up System (Promega; Thermo Fisher Scientific., Inc.). The DNA was eluted by $50 \mu 1$ of warm water and $5.5 \mu \mathrm{l}$ of $3 \mathrm{M} \mathrm{NaOH}$ were added at $37^{\circ} \mathrm{C}$ for $5 \mathrm{~min}$. The DNA was ethanol precipitated with glycogen as a carrier and resuspended in $20 \mu \mathrm{l}$ of water. Bisulfite-treated DNA was stored at $-20^{\circ} \mathrm{C}$ until further use.

Quantitative methylation-specific polymerase chain reaction (QMSP) for detecting differential methylation. The Multisource Genomic DNA Miniprep kit (Axygen Scientific, Inc. Union City, CA, USA) was used to extract total DNA from 60,000 cells/well, following the manufacturer's protocol. The CpGenome Universal DNA Modification kit (Chemicon International, Inc., Temecula, CA, USA) was used for DNA bisulfite modification. To determine the methylation status of mutL homolog (MLH), phosphatase and tensin homolog (PTEN), runt-related transcription factor (RUNX), Ras association domain family (RASSF), cadherin 1 (CDH1), O-6-methylguanine-DNA methyltransferase (MGMT) and P16. QMSP was performed in a TaqMan probe system using an Applied Biosystems 7900HT Fast Real-Time PCR System in a total volume of $20 \mu \mathrm{l}$ reaction mixture containing $2 \mu \mathrm{l}$ of bisulfite template DNA, $250 \mathrm{nM}$ of each primer (primer sequences of MLH, PTEN, RUNX, CDH1, MGMT and P16 are in Table I), $225 \mathrm{nM}$ TaqMan probe, and $10 \mu \mathrm{l}$ of FastStart Universal Probe Master (ROX; Roche Diagnostics, Roche Applied Science, Mannheim, Germany).

Analysis of differential gene transcription. Principal component analysis (PCA) was used to analyze data obtained from QMSP in which the content contains some inter-correlated quantitative dependent variables. Partek Genomics Suite 6.5 (Partek, Inc., St Louis, MO, USA) was used to analyze the gene expression data. The analyzed data were corrected and normalized by quantile normalization and summarization. PCA was used for global visualization of all data sets. 
Table I. Primer sequences.

\begin{tabular}{cl} 
Gene & \multicolumn{1}{c}{ Primer Sequences (5'-3') } \\
\hline MLH & \\
R & ATGGCCTGAATGGAGCCCCAGGAGAGG \\
F & TCCATTCAGGCCATCGCCTGTGCTGAG \\
PTEN & \\
R & TTTCATGGTGTTTTATCCCTC \\
F & TTTCCTGCAGAAAGACTTGA \\
RUNX & \\
R & CCTGACGAAGTGCCATAGTAGA \\
F & CCACCACTCACTACCACACCTA \\
RASSF & \\
R & TTTGTGAGAGTGTGTTTAGTTTTG \\
F & CCCAATTAAACCCATACTTCA \\
CDH1 & \\
R & TCCCCAAAACGAAACTAACGAC \\
F & AATTTTAGGTTAGAGGGTTATCGCGT \\
MGMT & \\
R & CAACATCACTAACACCTAACC \\
F & CCTAATGTTGGGATAGTT \\
P16 & \\
R & ACCCGACCCCGAACCGCGACCGTAA \\
F & TTATTAGAGGGTGGGGCGGATCGCGTCG
\end{tabular}

MLH, mutL homolog; PTEN, phosphatase and tensin homolog; RUNX, runt-related transcription factor; RASSF, Ras association domain family; CDH1, cadherin 1; MGMT, O-6-methylguanine-DNA methyltransferase; F, forward; $\mathrm{R}$, reverse.

Functional enrichment and pathway enrichment analysis. The Database for Annotation, Visualization and Integrated Discovery (DAVID) online tool was used to conduct Gene Ontology (GO; www. geneontology. org/) and KEGG (Kyoto Encyclopedia of Genes and Genomes) pathway enrichment analysis (www. genome. jp/kegg/). DAVID is used to convert collected data into biological meaning and contributes to the explanation of data sets on a genome-wide scale. GO terms and KEGG pathways of which $\mathrm{P}<0.1$ were chosen as previously described (20).

Statistical analysis. SPSS 18.0 (SPSS, Inc., Chicago, IL, USA) and GraphPad Prism 6 (GraphPad Software, Inc., La Jolla, CA, USA) were used to analyze the data. A two-tailed $\mathrm{t}$-test was used to differentiate the mean methylation scores between two samples. A paired t-test and one-way analysis of variance were applied to determine the differences of average sib pair in methylation scores. The network representation was generated using GeneGO MetaCore software (version 4.3; www.genego.com/.metacore.php, GeneGo,Inc., Encinitas, CA, USA). The Venn diagram is a graphic organizer constructed by overlapping circles to indicate features common or unique to two or more concepts (21). Methylation diversity was obtained by Genomestudio software version 2010.1.

\section{Results}

Analysis of differential gene transcription. Partek Genomics Suite 6.5 (Partek, Inc.) was used to analyze the gene expression data. The data were subsequently corrected and normalized by quantile normalization and summarization. Cells were cultured at 2D, 3D and Ti substrate. PCA was used for global visualization of all data, which revealed the close connections between the 3D, 2D and Ti groups (Fig. 1A). Gene lists were established with a $\mathrm{P} \leq 0.05$. A Venn diagram of bisulfite modification DNA in 3D group, 2D group and Ti group was created (Fig. 1B). The results revealed that 116 genes were common among the 231-3D vs. 231-2D group, 231-3D vs. 231-Ti group and 231-Ti vs. 231-2D group. Other than the 116 common genes, 152 genes were shared in the 231-3D vs. 231-2D group and the 231-Ti vs. 231-2D group, 109 genes were common in the $231-3 \mathrm{D}$ vs. $231-2 \mathrm{D}$ group and the $231-3 \mathrm{D}$ vs. 231-Ti group, and 80,311 genes were common in the $231-3 \mathrm{D}$ vs. 231-Ti group and the 231-Ti vs. 231-2D group. Unsupervised clustering analysis of the $\mathrm{CpG}$ location indicated that $268 \mathrm{CpGs}$ presented different levels of methylation in the three groups of samples, and $116 \mathrm{CpG}$ were highly methylated in the three groups of samples (Fig. 1C).

Analysis on different $C p G$ location. To analyze the different CpG locations in breast cancer cells cultured in 2D, 3D and Ti substrates, scatter plots were prepared that compared the CpG sites in 231-3D, 231-2D and 231-Ti group (Fig. 2). The methylation patterns between $231-3 \mathrm{D}$ vs. $231-2 \mathrm{D}$ (Fig. 2A) are more similar compared with those between 231-3D vs. 231-Ti (Fig. 2B) or 231-Ti vs. 231-2D (Fig. 2C). Fig. 2D-F displays the column distribution of $\beta$-values in the three groups of samples.

Functional analysis of methylated DNA. To identify the biological functions, cellular components and molecular functions in which the identified genes may serve a role, GO term analysis was performed. GO term analysis identified numerous genes that were expressed differentially in different cellular processes, including signal transduction, transmembrane transport, cell differentiation, sequence-specific DNA binding transcription factor activity, sequence-specific-DNA binding, G-protein coupled receptor activity and transporter activity (Fig. 3A). To further refine the biological functions of genes corresponding to differential methylation, KEGG enriched pathway analysis was used to systematically analyze gene functions based on networks of genes and molecules. Pathway analyses of the corresponding genes identified 14 significantly over-represented cellular pathways (Fig. 3B), of which 8 were more significant, including pathways in cancer, mitogen-activated protein kinase (MAPK) signaling pathway, regulation of actin cytoskeleton, intestinal immune network for immunoglobulin (Ig)A production, Staphylococcus aureus infection, neuroactive ligand-receptor interaction, type 1 diabetes mellitus and Notch signaling pathway. To determine whether the different cell culture methods effected methylation of important genes, QMSP analysis was used. To determine whether the different cell culture methods have an effect on genomic DNA methylation, the methylation levels of MLH, PTEN, RUNX, RASSF, CDH1, MGMT and P16 genes was investigated in breast cancer cell lines MCF-7 cultured 
A

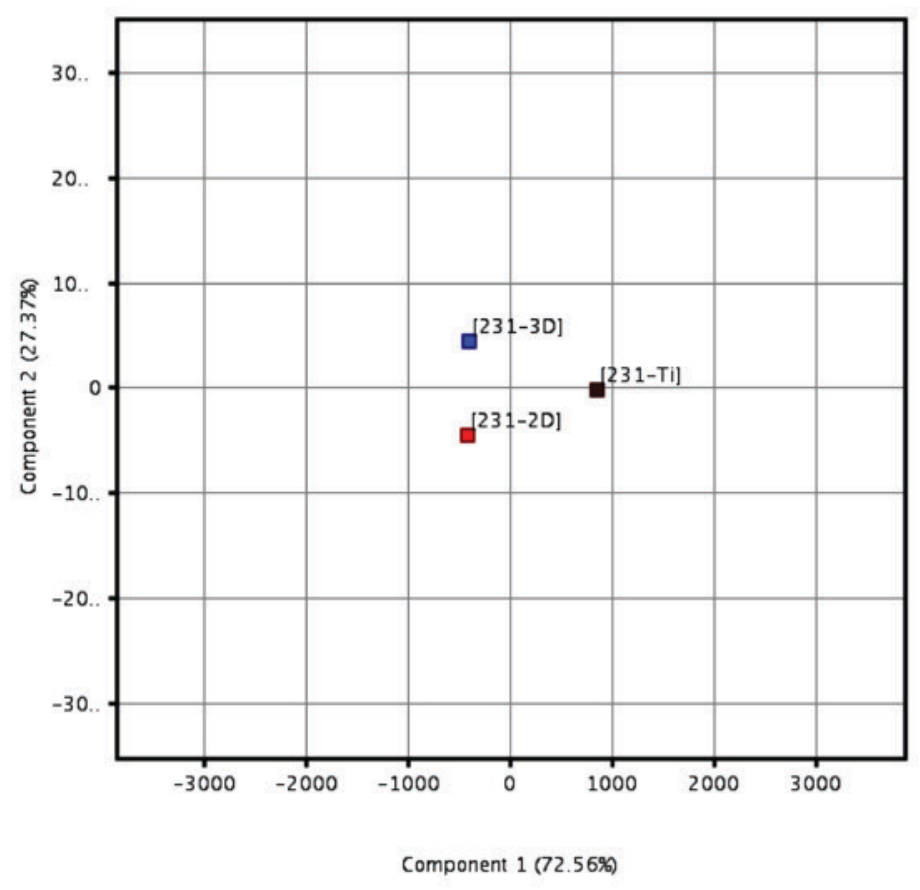

C

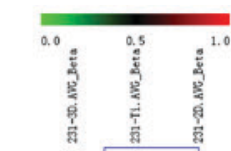

B

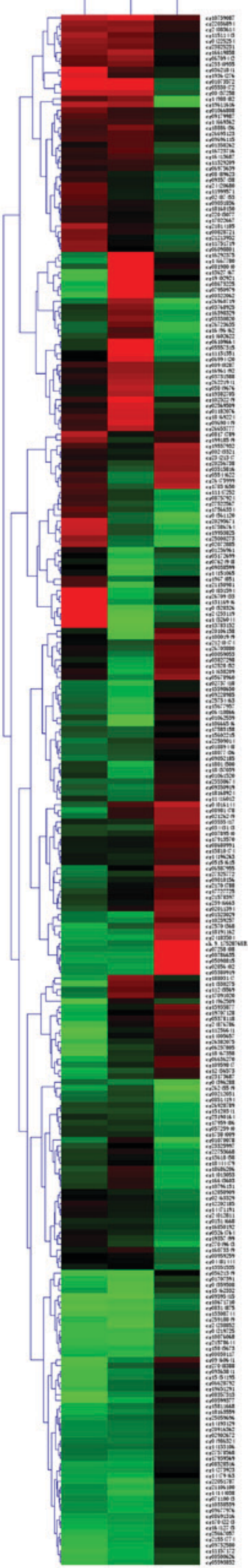

Figure 1. Hypermethylated genes are found in breast cancers cultured under 2D, 3D and Ti conditions. (A) Principal Components Analysis. The 231-3D, 231-2D and 231-Ti data sets were analyzed to study the association of differential methylation DNA among 3 culture conditions. (B) Venn diagram was used to identify differential methylation sites in 3 culture conditions. (C) Unsupervised clustering analysis of the $\mathrm{CpG}$ location. The color gradient green to red indicates the $\beta$-value $(0-1)$. D, dimensional; Ti, orthotopic transplantation. 
A

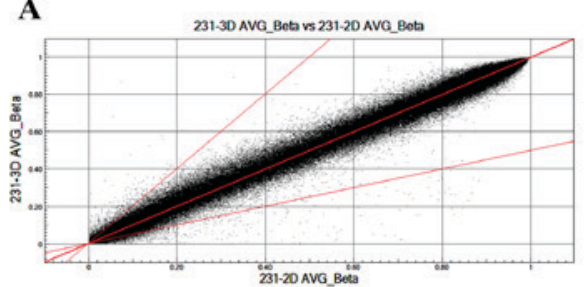

D

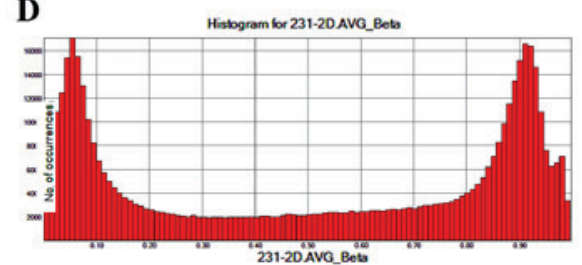

B

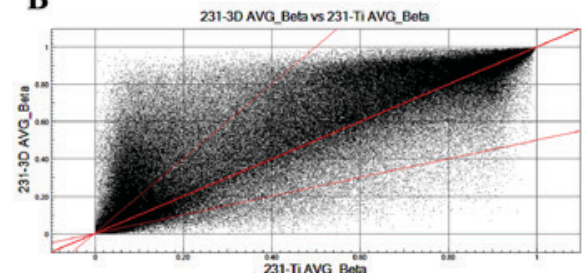

E

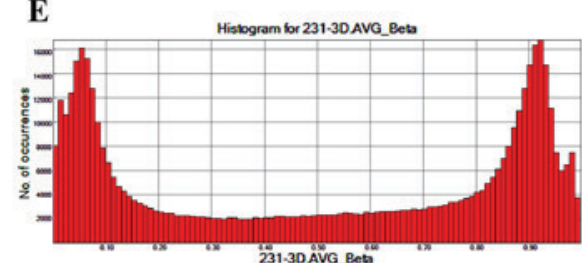

C
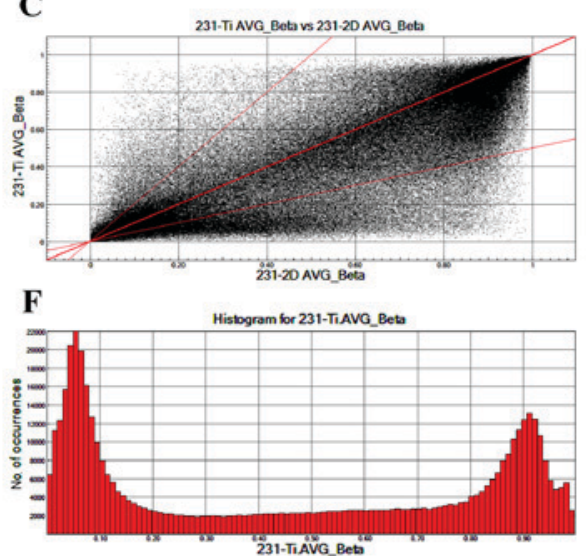

Figure 2. Methylation pattern in 231-3D vs. 231-2D, 231-3D vs. 231-Ti and 231-Ti vs. 231-2D were not significantly different. (A-C) Scatter plot of average value of beta values in 3D vs. 2D, 3D vs. Ti and Ti vs. 2D data. (D-F) Column distribution of $\beta$-values in 3 culture conditions. D, dimensional; Ti, orthotopic transplantation.

A

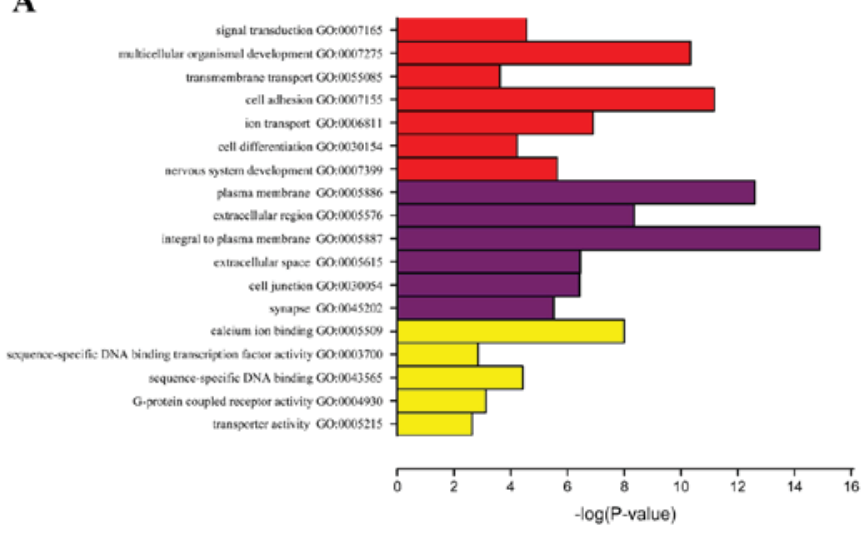

B

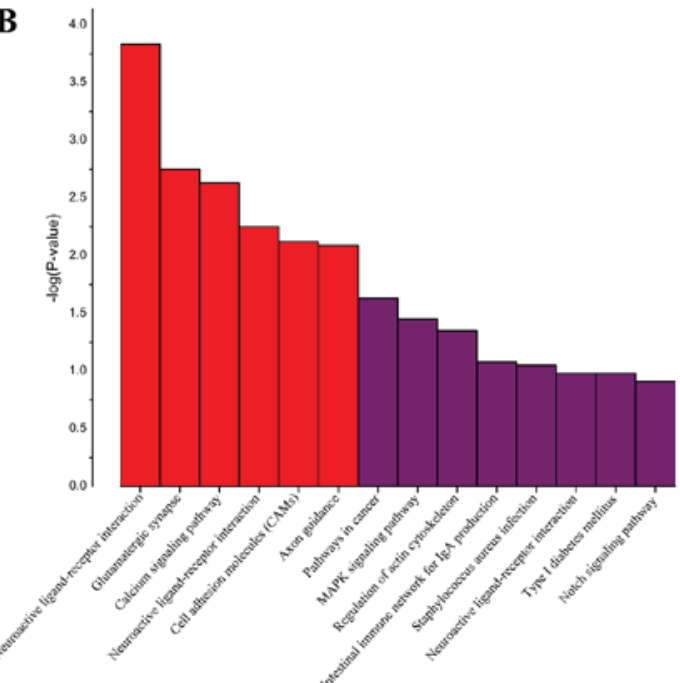

Figure 3. (A) GO analysis of corresponding genes with different methylation sites. (B) KEGG pathway analysis of the corresponding genes with different methylation sites. GO, gene ontology; KEGG, Kyoto Encyclopedia of Genes and Genomes.

in 2D, 3D or Ti culture pattern, respectively. By methylation analysis, methylation levels of MLH, PTEN, RUNX, RASSF, CDH1, MGMT and P16 genes had no significant difference between 2D, 3D and Ti culture pattern (data not shown).

\section{Discussion}

DNA hypermethylation is reported to serve a role in many cancers such as breast cancer. During the progression of cancer, hypermethylation of $\mathrm{CpG}$ islands serves a key role in silencing tumor regulatory genes (22). The observation of epigenetic changes indicated that DNA hypermethylation is a key factor influencing the progression of breast cancer (23). DNA hypermethylation often occurs in cancer cells and specific sets of genes (24).

PCA is a very useful method to analyze data tables, and data are expressed with some inter-correlated quantitative dependent variables (25). Previous studies have used PCA to extract information and to display the pattern of similarity between the number of variables and the number of observations in PCA maps (26). In the present study, PCA was used to analyze the methylation differences of DNA in MCF-7 breast cancer cells under 2D, 3D or Ti adhesive substrate culturing conditions. The results indicated that the differentially methylated DNA in the three groups was closely related with each other. In addition, a total of 116 differentially methylated sites were identified as commonly occurring in the 3 groups of samples, and the common sections presented high rates of methylation. Unsupervised clustering analysis was used to explore the methylation status of CpGs. The results demonstrated that $268 \mathrm{CpGs}$ presented different levels of methylation in the three groups of samples and $116 \mathrm{CpGs}$ of which all appeared high methylation status. Abnormal DNA methylation often occurs in $\mathrm{CpG}$ islands, and $\mathrm{CpG}$ island shores serve a key role in harboring the changes of DNA methylation (27). In breast cancer, methylation of $\mathrm{CpG}$ island shores is associated with clinical features (28). Scatter plots comparing all CpG sites among the 231-3D, 231-2D and 231-Ti groups 
were constructed to analyze the different $\mathrm{CpG}$ locations in breast cancer cells cultured in 2D, 3D and Ti substrate. The results revealed that the methylation pattern between 231-3D vs. 231-2D, 231-3D vs. 231-Ti and 231-Ti vs. 231-2D was no significant different.

$\mathrm{GO}$ analysis results indicated that genes with differential expression are involved in different cellular processes, such as signal transduction, transmembrane transport, cell differentiation, sequence-specific DNA binding transcription factor activity, sequence-specific-DNA binding, G-protein coupled receptor activity and transporter activity. Abnormalities in PTEN, k-RAS, or $\beta$-catenin genes can alter several different signal transduction pathways (29). RASSF play an important role in transmembrane transport. They are Ras effectors and are transported into the nucleus by classical nuclear transport pathways (30). CDH1 is involved in cell differentiation and has a capacity to control cell fate by altering directional cell proliferation and apoptosis (31). KEGG results demonstrated that genes were enriched in 14 pathways including pathways in cancer, MAPK signaling pathway, Regulation of actin cytoskeleton, Intestinal immune network for IgA production, Staphylococcus aureus infection, Neuroactive ligand-receptor interaction, Type 1 diabetes mellitus and Notch signaling pathway. Therefore, the role of different culture methods on the methylated level of MLH, PTEN, RUNX, RASSF, CDH1, MGMT and P16 gene was investigated, via QMSP and demonstrated that the was no significant difference between 2D, 3D and Ti culture pattern. PTEN suppresses tumor development and metastasis, and is mutated in many cancers (32). Abnormal expression of PTEN was observed in many tumors (33). In breast cancer, PTEN inhibits cell growth and induces apoptosis (34). RUNX genes have attracted increasing attention, owing to their roles in suppressing or promoting tumors (35). In many key pathways, RUNX may regulate lineage-specific gene expression $(27,36)$. RUNX family members may serve a broader role in multistep breast tumorigenesis (37). In the progression of many cancers, RASSF proteins are often downregulated and may suppress tumor development and metastasis (38). RASSF gene is often silenced by promoter methylation (39). The present study demonstrated that methylation of MLH, PTEN, RUNX, RASSF, CDH1, MGMT and P16 genes had no difference under 2D, 3D or Ti culture conditions.

In conclusion, changes in methylation status may be associated with the occurrence and metastasis of breast cancer. Growth environment of breast cancer cells have no influence on methylation status of MLH, PTEN, RUNX, RASSF, CDH1, MGMT and P16 genes. The present findings may shed light to treating breast cancer by identifying known and novel gene targets.

\section{References}

1. Ferlay J, Shin HR, Bray F, Forman D, Mathers C and Parkin DM: Estimates of worldwide burden of cancer in 2008: GLOBOCAN 2008. Int J Cancer 127: 2893-2917, 2010.

2. van 't Veer LJ, Dai H, van de Vijver MJ,He YD, Hart AA, Mao M, Peterse HL, van der Kooy K, Marton MJ, Witteveen AT, et al: Gene expression profiling predicts clinical outcome of breast cancer. Nature 415: 530-536, 2002.

3. Li S, Rong M and Iacopetta B: DNA hypermethylation in breast cancer and its association with clinicopathological features. Cancer Lett 237: 272-280, 2006.
4. Yegnasubramanian S, Haffner MC, Zhang Y, Gurel B, Cornish TC, Wu Z, Irizarry RA, Morgan J, Hicks J, DeWeese TL, et al: DNA hypomethylation arises later in prostate cancer progression than $\mathrm{CpG}$ island hypermethylation and contributes to metastatic tumor heterogeneity. Cancer Res 68: 8954-8967, 2008.

5. Ji H, Ehrlich LI, Seita J, Murakami P, Doi A, Lindau P, Lee H, Aryee MJ, Irizarry RA, Kim K, et al: Comprehensive methylome map of lineage commitment from haematopoietic progenitors. Nature 467: 338-342, 2010.

6. Doi A, Park IH, Wen B, Murakami P, Aryee MJ, Irizarry R, Herb B, Ladd-Acosta C, Rho J, Loewer S, et al: Differential methylation of tissue- and cancer-specific $\mathrm{CpG}$ island shores distinguishes human induced pluripotent stem cells, embryonic stem cells and fibroblasts. Nat Genet 41: 1350-1353, 2009.

7. Portela A and Esteller M: Epigenetic modifications and human disease. Nat Biotechnol 28: 1057-1068, 2010.

8. Tsai HC and Baylin SB: Cancer epigenetics: Linking basic biology to clinical medicine. Cell Res 21: 502-517, 2011.

9. Fackler MJ, McVeigh M, Evron E, GarrettE, Mehrotra J, Polyak K, Sukumar S and Argani P: DNA methylation of RASSF1A, HIN-1, RAR-beta, Cyclin D2 and Twist in in situ and invasive lobular breast carcinoma. Int J Cancer 107: 970-975, 2003.

10. Dimas AS, Deutsch S, Stranger BE, Montgomery SB, Borel C, Attar-Cohen $\mathrm{H}$, Ingle $\mathrm{C}$, Beazley $\mathrm{C}$, Gutierrez Arcelus M, Sekowska M, et al: Common regulatory variation impacts gene expression in a cell type-dependent manner. Science 325: 1246-1250, 2009.

11. Murrell A, Heeson S, Cooper WN, Douglas E, Apostolidou S, Moore GE, Maher ER and Reik W: An association between variants in the IGF2 gene and Beckwith-Wiedemann syndrome: Interaction between genotype and epigenotype. Hum Mol Genet 13: 247-255, 2004.

12. Kerkel K, Spadola A, Yuan E, Kosek J, Jiang L, Hod E, Li K, Murty VV, Schupf N, Vilain E, et al: Genomic surveys by methylation-sensitive SNP analysis identify sequence-dependent allele-specific DNA methylation. Nat Genet 40: 904-908, 2008.

13. Li XJ, Valadez AV, Zuo P and Nie Z: Microfluidic 3D cell culture: Potential application for tissue-based bioassays. Bioanalysis 4: 1509-1525, 2012.

14. Baharvand H, Hashemi SM, Kazemi Ashtiani S and Farrokhi A: Differentiation of human embryonic stem cells into hepatocytes in 2D and 3D culture systems in vitro. Int J Dev Biol 50: 645-652, 2006.

15. Tibbitt MW and Anseth KS: Hydrogels as extracellular matrix mimics for 3D cell culture. Biotechnol Bioeng 103: 655-663, 2009.

16. Yu M, Huang S, Yu KJ and Clyne AM: Dextran and polymer polyethylene glycol (PEG) coating reduce both 5 and $30 \mathrm{~nm}$ iron oxide nanoparticle cytotoxicity in $2 \mathrm{D}$ and $3 \mathrm{D}$ cell culture. Int $\mathrm{J}$ Mol Sci 13: 5554-5570, 2012.

17. Weigelt B, Ghajar CM and Bissell MJ: The need for complex 3D culture models to unravel novel pathways and identify accurate biomarkers in breast cancer. Adv Drug Deliv Rev 69-70: 42-51, 2014.

18. Hájková H, Fritz MH, Haškovec C, Schwarz J, Šálek C, Marková J, Krejčík Z, Dostálová Merkerová M, Kostečka A, Vostrý M, et al: CBFB-MYH11 hypomethylation signature and PBX3 differential methylation revealed by targeted bisulfite sequencing in patients with acute myeloid leukemia. J Hematol Oncol 7: 66, 2014.

19. Liu X, Jia X, Yuan H, Ma K, Chen Y, Jin Y, Deng M, Pan W, Chen S, Chen Z, et al: DNA methyltransferase 1 functions through C/ebpa to maintain hematopoietic stem and progenitor cells in zebrafish. J Hematol Oncol 8: 15, 2015.

20. Ma CH, Lv Q, Cao Y, Wang Q, Zhou XK, Ye BW and Yi CQ: Genes relevant with osteoarthritis by comparison gene expression profiles of synovial membrane of osteoarthritis patients at different stages. Eur Rev Med Pharmacol Sci 18: 431-439, 2014.

21. Chen $\mathrm{H}$ and Boutros PC: VennDiagram: A package for the generation of highly-customizable Venn and Euler diagrams in R. BMC Bioinformatics 12: 35, 2011.

22. Jones PA and Baylin SB: The fundamental role of epigenetic events in cancer. Nat Rev Genet 3: 415-428, 2002.

23. Lewis CM, Cler LR, Bu DW, Zöchbauer-Müller S, Milchgrub S, Naftalis EZ, Leitch AM, Minna JD and Euhus DM: Promoter hypermethylation in benign breast epithelium in relation to predicted breast cancer risk. Clin Cancer Res 11: 166-172, 2005. 
24. Stirzaker C, Song JZ, Davidson B and Clark SJ: Transcriptional gene silencing promotes DNA hypermethylation through a sequential change in chromatin modifications in cancer cells. Cancer Res 64: 3871-3877, 2004.

25. Abdi $\mathrm{H}$ and Williams LJ: Principal component analysis. Wiley Interdiscip Rev Comput Stat 2: 433-459, 2010.

26. Bro R and Smilde AK: Principal component analysis. Anal Methods 6: 2812-2831,2014.

27. Irizarry RA, Ladd-Acosta C, Wen B, Wu Z, Montano C, Onyango P, Cui H, Gabo K, Rongione M, Webster M, et al: The human colon cancer methylome shows similar hypo- and hypermethylation at conserved tissue-specific $\mathrm{CpG}$ island shores. Nat Genet 41: 178-186, 2009.

28. Farkas SA, Milutin-Gašperov N, Grce $M$ and Nilsson TK: Genome-wide DNA methylation assay reveals novel candidate biomarker genes in cervical cancer. Epigenetics 8: 1213-1225, 2013.

29. Matias-Guiu X, Catasus L, Bussaglia E, Lagarda H, Garcia A, Pons C, Muñoz J, Argüelles R, Machin P and Prat J: Molecular pathology of endometrial hyperplasia and carcinoma. Hum Pathol 32: 569-577, 2001.

30. Kumari G, Singhal PK, Rao MR and Mahalingam S: Nuclear transport of Ras-associated tumor suppressor proteins: Different transport receptor binding specificities for arginine-rich nuclear targeting signals. J Mol Biol 367: 1294-1311, 2007.

31. Reardon SN, King ML, MacLean JA II, Mann JL, DeMayo FJ, Lydon JP and Hayashi K: CDH1 is essential for endometrial differentiation, gland development, and adult function in the mouse uterus. Biol Reprod 86: 141, 1-10, 2012

32. Simpson L and Parsons R: PTEN: Life as a tumor suppressor. Exp Cell Res 264: 29-41, 2001.
33. Maehama T and Dixon JE: PTEN: A tumour suppressor that functions as a phospholipid phosphatase. Trends Cell Biol 9: $125-128,1999$.

34. Stemke-Hale K, Gonzalez-Angulo AM, Lluch A, Neve RM, Kuo WL, Davies M, Carey M, Hu Z, Guan Y, Sahin A, et al: An integrative genomic and proteomic analysis of PIK3CA, PTEN and AKT mutations in breast cancer. Cancer Res 68: 6084-6091, 2008.

35. Blyth K, Cameron ER and Neil JC: The RUNX genes: Gain or loss of function in cancer. Nat Rev Cancer 5: 376-387, 2005.

36. Levanon D and Groner Y: Structure and regulated expression of mammalian RUNX genes. Oncogene 23: 4211-4219, 2004.

37. Lau QC, Raja E, Salto-Tellez M, Liu Q, Ito K, Inoue M, Putti TC, Loh M, Ko TK, Huang C, et al: RUNX3 is frequently inactivated by dual mechanisms of protein mislocalization and promoter hypermethylation in breast cancer. Cancer Res 66: 6512-6520, 2006.

38. Allen NP, Donninger H, Vos MD, Eckfeld K, Hesson L, Gordon L, Birrer MJ, Latif $\mathrm{F}$ and Clark GJ: RASSF6 is a novel member of the RASSF family of tumor suppressors. Oncogene 26: 6203-6211, 2007.

39. Djos A, Martinsson T, Kogner P and Carén H: The RASSF gene family members RASSF5, RASSF6 and RASSF7 show frequent DNA methylation in neuroblastoma. Mol Cancer 11: 40, 2012. 